\title{
Anabases
}

ANABASES Traditions et réceptions de l'Antiquité

5 | 2007

Varia

\section{L'« inventaire des différences » en couleurs : à la recherche du paysage sensible des Grecs à l'époque archaïque}

Adeline Grand-Clément

\section{OpenEdition}

Journals

Édition électronique

URL : http://journals.openedition.org/anabases/3227

DOI : $10.4000 /$ anabases.3227

ISSN : 2256-9421

Éditeur

E.R.A.S.M.E.

Édition imprimée

Date de publication : 1 mars 2007

Pagination : 249-256

ISSN : $1774-4296$

Référence électronique

Adeline Grand-Clément, «L'« inventaire des différences » en couleurs : à la recherche du paysage sensible des Grecs à l'époque archaïque », Anabases [En ligne], 5 | 2007, mis en ligne le 01 janvier 2012, consulté le 21 octobre 2019. URL : http://journals.openedition.org/anabases/3227 ; DOI : 10.4000/anabases.3227

Ce document a été généré automatiquement le 21 octobre 2019

(c) Anabases 


\title{
L'« inventaire des différences » en couleurs : à la recherche du paysage sensible des Grecs à l'époque archaïque
}

\author{
Adeline Grand-Clément
}

Pourquoi les Égyptiens, experts en l'art de manier les couleurs, comme en témoignent encore aujourd'hui leurs palettes à fard et les parois richement colorées de leurs tombes, donnaient-ils à la mer Méditerranée le nom de Ouadj-Ouret, «Grande Verte »? L'étendue marine, si elle possède des teintes diverses, nous apparaît fondamentalement bleue. Plus intrigant encore - car les Grecs nous semblent plus proches, plus familiers que les Égyptiens - dans les épopées homériques, la mer tumultueuse qui blanchit les rivages d'Ithaque ou la proue du navire d'Ulysse inquiète par sa teinte "pourpre"

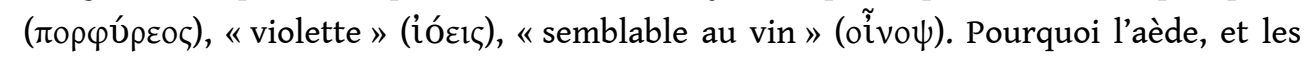
poètes grecs à sa suite, ne vantent-ils pas l'azur des flots méditerranéens? Une telle question, qui n'a pas manqué de retenir l'attention de certains savants dès le XIX ${ }^{e}$ siècle ${ }^{1}$, invite à admettre que la perception des couleurs varie en fonction des cultures et que les faits de langue traduisent ces différences. Dès lors, la question du chromatisme peut légitimement participer à dresser cet «inventaire des différences» qui, selon Paul Veyne, sous-tend le travail de l'historien:

L'histoire peut être définie comme l'inventaire explicatif [...] de ce qu'il y a de social en l'homme, ou plus précisément des différences que présente cet aspect social. Il suffit par exemple que la perception des couleurs soit différente d'une société à l'autre (aux yeux des Grecs, la mer était violette); ipso facto, les couleurs appartiendront à l'histoire autant qu'à la science ${ }^{2}$.

2 Étudier la réception des couleurs à l'époque archaïque grecque, comme nous nous sommes proposée de le faire dans le cadre d'une thèse de doctorat ${ }^{3}$, permet ainsi de pénétrer d'une façon neuve dans la culture et l'imaginaire des Anciens, en cernant les principaux traits de leur altérité. 


\section{Généalogie d'un " problème » : les Grecs et la couleur. Vers une étude du paysage sensible}

3 De prime abord, le rapport que les Grecs entretenaient avec le phénomène chromatique semble relever de la schizophrénie. Muets à propos de certaines couleurs, qu'ils ne nommaient pas, ils les utilisaient en abondance pour décorer leurs statues et leurs édifices: voilà la conclusion naturelle à laquelle conduisait l'état de la recherche jusqu'à une date très récente. Au $\mathrm{XIX}^{\mathrm{e}}$ siècle, William Gladstone, un spécialiste d'Homère, par ailleurs grand homme d'État britannique, ainsi qu'Hugo Magnus, un ophtalmologiste de Breslau, tous deux séduits par les théories évolutionnistes alors en vigueur, avaient conclu à un daltonisme des Grecs, affirmant que leur vision des couleurs était déficiente ${ }^{4}$. Ils se fondaient principalement sur une analyse du vocabulaire des couleurs dans les épopées homériques, davantage tourné, selon eux, vers les contrastes entre le clair et le sombre, la lumière et l'obscurité. À la thèse d'une forme d'immaturité visuelle des Grecs, rapidement abandonnée, s'est aussitôt substituée l'idée d'un désintérêt, d'une insensibilité à l'égard du phénomène chromatique. Elle est restée aujourd'hui vivace dans les travaux de certains philologues, étonnés par les étrangetés du lexique grec des couleurs, en particulier l'absence troublante $\mathrm{du}$ « bleu » et du « vert».

D'un autre côté, l'ouverture de la Grèce aux voyageurs, architectes et archéologues, à partir du début du XIX $x^{e}$ siècle, a permis au milieu savant européen de découvrir les sculptures et les monuments grecs. Les travaux d'Antoine Quatremère de Quincy et de Jacques-Ignace Hittorff, ainsi que les publications de plusieurs archéologues anglais ou allemands, ont contraint les hellénistes à admettre, parfois contre leur gré, la nature profondément polychrome de l'art grec ${ }^{5}$. La découverte ébranlait les fondements de l'idéal classique défini par Winckelmann ${ }^{6}$, qui avait consacré le triomphe de la blanche lumière, au détriment du chatoiement, plaisant mais trompeur, des couleurs. On considérait alors que les teintes douces ne pouvaient être appréciées que par des yeux d'hommes civilisés, éduqués, sensibles à la noblesse sobre et élégante du dessin et de la forme. Les "primitifs", eux, raffolaient, à l'instar des femmes et des enfants, de violents contrastes chromatiques. Ce sont de tels présupposés qui font écrire à Henri Lechat, spécialiste de la sculpture grecque :

Pour des hommes demi-civilisés la forme isolée, toute nue, ne satisfait point les yeux; ils ne la comprennent que sous le vêtement de la couleur. L'idole, peinte et parée, produit sur eux une impression plus vive ${ }^{7}$.

L'usage de la polychromie constituait donc un signe d'immaturité. La vivacité des contrastes chromatiques, encore visibles sur certains vestiges de sculpture, s'expliquait par l'« archaïsme » des œuvres concernées, réalisées par des artisans ne maitrisant pas encore la virtuosité, la finesse d'exécution des maîtres du classicisme.

6 On ne peut plus soutenir aujourd'hui l'idée que l'engouement des Grecs pour la polychromie est à mettre au compte de leur état "primitif", d'une sensibilité chromatique immature, symptôme d'un stade de développement encore imparfait, dont témoignerait également le lexique. L'un des premiers à avoir tenté de retrouver la spécificité du regard grec sur la couleur, en s'affranchissant des catégories et des jugements modernes, fut Louis Gernet. En 1957, à la demande d'Ignace Meyerson, il a contribué à l'ouvrage collectif Problèmes de la couleur, qui envisageait la question de la dénomination et de la perception des couleurs sous l'angle du comparatisme. Dans 
l'article qu'il consacrait à ce problème anthropologique chez les Grecs, Louis Gernet jugeait que «rien n'atteste [...] dans le vocabulaire qu'il y ait eu enrichissement ou variation de la sensibilité aux couleurs depuis le plus ancien témoignage qui est celui d'Homère "; bien au contraire, "dès le début et de façon constante, cette sensibilité s'affirme spécialement ${ }^{8} »$. L'auteur invitait à replacer le fait linguistique dans sa

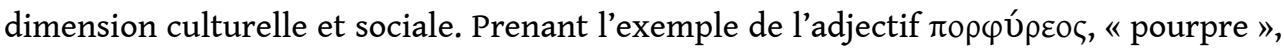
et de l'utilisation de cette teinture prestigieuse, il montrait comment une étude consacrée aux termes chromatiques pouvait, lorsqu'elle était mise en regard avec la question des pratiques, enrichir notre connaissance et notre compréhension des systèmes symboliques qui régissaient l'univers des Grecs ${ }^{9}$.

7 Depuis, les travaux de l'historien médiéviste et anthropologue Michel Pastoureau ont approfondi les pistes qui permettent d'explorer les voies de la « différence » grecque. Dans une stimulante Histoire du Bleu, l'auteur a montré le complet renversement de la place accordée à la couleur bleue dans les sociétés humaines, depuis le néolithique jusqu'au $\mathrm{xx}^{\mathrm{e}}$ siècle, mettant l'accent sur le changement qui intervient au cours du Moyen Âge. L'étude a prouvé qu'il était pertinent d'envisager la couleur comme un objet historique à part entière. Apprivoisée, façonnée par des pratiques, la couleur s'inscrit dans l'horizon des représentations mentales d'une société donnée. L'étude de l'accueil réservé aux couleurs permet de sonder le lieu où affleurent, en interférant mutuellement, les manières de sentir, de penser et d'agir : c'est ce lieu que nous avons baptisé le "paysage sensible». La formule renvoie aux travaux d'Alain Corbin, historien des époques moderne et contemporaine, attentif aux "dispositifs affectifs » habituellement délaissés par les chercheurs ${ }^{10}$. Choisissant des objets d'étude en apparence anodins ou marginaux, tels les parfums et les odeurs, le son des cloches dans les campagnes, il a tenté de restituer la configuration des sensibilités collectives des sociétés européennes des XVIII ${ }^{\mathrm{e}}$ et $\mathrm{XIX}^{\mathrm{e}}$ siècles ${ }^{11}$. De la même manière, les couleurs, longtemps écartées de l'histoire grecque, peuvent elles aussi constituer un terrain d'investigation fécond, susceptible d'offrir une vision cohérente des systèmes de représentation et d'appréciation tissés au sein de la société grecque archaïque.

\section{Rassembler et croiser les témoignages}

8 Le projet implique, comme le suggère Michel Pastoureau ${ }^{12}$, de prendre en compte tous les domaines de la couleur historiquement observables, c'est-à-dire de réunir l'ensemble des témoignages disponibles pour la période archaïque. Étudier conjointement les textes et les images, tenter de faire dialoguer les mots, les pratiques et les représentations, nécessite de confronter, de mettre en regard deux grands dossiers documentaires, l'un, littéraire, l'autre, archéologique ${ }^{13}$. Le premier se présente sous la forme d'un corpus relativement cohérent, regroupant l'ensemble des textes composés entre le viII siècle et la première moitié du v $v^{e}$ siècle, depuis les poèmes homériques et hésiodiques jusqu'au théâtre d'Eschyle. L'examen soigneux de ce dossier permet de nourrir une analyse détaillée du vocabulaire des couleurs. Une telle démarche est en revanche inenvisageable dans le cas de la documentation fournie par l'archéologie : le foisonnement et la diversité des œuvres présentant des vestiges de polychromie - vases, figurines, statues, reliefs, éléments architecturaux ${ }^{14}-$ ne permettent pas de mener l'enquête d'une façon exhaustive. La fragilité des pigments appliqués sur les objets, leur propension à s'évanouir ou à s'altérer - quand ils n'ont pas 
été soigneusement enlevés lors des nettoyages méticuleux effectués par les premiers découvreurs - compliquent les travaux d'interprétation et les tentatives de restitution de l'état originel ${ }^{15}$.

Lorsque Lucien Febvre, en 1941, invitait à écrire une histoire des sensibilités, dans un article intitulé "La sensibilité et l'histoire. Comment reconstituer la vie affective d'autrefois?", il n'hésitait pas à reconnaître que "prétendre reconstituer la vie affective d'une époque donnée, c'est une tâche à la fois extrêmement séduisante et affreusement difficile ${ }^{16} \%$. La difficulté s'accroît lorsque l'on étudie des sociétés anciennes. En effet, comment connaître la réception des couleurs dans une culture dont des pans entiers nous demeurent pratiquement inconnus - le domaine des tissus et du vêtement, par exemple, l'un des principaux vecteurs de la couleur? Le caractère lacunaire de la documentation ne constitue pas le seul obstacle auquel se heurte l'historien des sensibilités: les commanditaires ou les destinataires des textes, des images et des objets conservés pour la période archaïque appartiennent essentiellement aux couches les plus aisées de la société. Les sources reflèteraient-elles uniquement les goûts d'un groupe dominant, celui des cercles aristocratiques, laissant dans l'ombre ceux du reste de la population? Fort heureusement, la publicité offerte, au sein des cités, aux productions littéraires, largement diffusées lors des représentations orales, et artistiques, souvent exposées aux yeux de tous, pallie pour une bonne part le biais de la documentation. La fonction éminemment sociale que possédaient la poésie et les arts visuels, dans la Grèce archaïque, incite également à porter une attention soutenue aux contextes au sein desquels le chant poétique ou l'objet polychrome prenaient place. Les deux modes d'expression permettaient de véhiculer des valeurs, contribuaient à façonner les systèmes de représentation et d'appréciation du groupe - celui des convives de banquet, du cercle familial réuni lors des mariages ou des funérailles, de l'ensemble de la communauté civique rassemblée lors des festivités annuelles. La part que prenaient les couleurs dans ce processus était loin d'être négligeable: les signes chromatiques servaient à classer, identifier, hiérarchiser. Leur histoire révèle ainsi la tension qui existait, au sein de la cité archaïque, entre les efforts constants menés par les aristocrates pour se distinguer, prouver leur excellence, et les préoccupations de la communauté, soucieuse de mettre " au milieu », es meson, des valeurs et une identité partagées par l'ensemble du groupe.

\section{En tirant les fils de couleurs...}

L'enquête a pris pour point de départ une anecdote relatée par Ion de Chios, qui invite à considérer, comme le suggérait Louis Gernet, que les Grecs, dès le milieu du ve siècle, étaient conscients de l'existence de deux langages de la couleur distincts, celui de la peinture, "où il est postulé que les couleurs sont les "vraies" ", et celui de la poésie, " où l'adjectif répond bien plutôt à un sentiment qu'à une perception ${ }^{17}$ ". En apparence inconciliables, ces deux versants de l'accueil réservé par les Grecs aux couleurs, renvoyant dos à dos la figure du poète et celle de l'artisan, ne sont-ils cependant pas susceptibles de révéler des façons de sentir et de penser analogues - du moins pour ce qui concerne l'époque archaïque?

11 Un premier parcours au sein des textes archaïques a permis de cerner les notions de «couleur» et de "coloration", en sondant les catégories antiques. Les Grecs percevaient d'abord la couleur comme une surface, inséparable de son support, non 
comme une donnée abstraite. La peau de l'homme servait de modèle de référence, comme en témoigne l'histoire du terme $\chi \rho \omega ́ \varsigma$, dont dérive $\chi \rho \tilde{\omega} \mu \alpha$, mot qui désigne spécifiquement la couleur, à partir de la période classique. L'épiderme humain représentait la surface colorée par excellence, fragile, délicate, sujette au changement, comme la fleur menacée de flétrissement. La métamorphose et le mouvement participaient pleinement à construire la notion de "couleur/coloration ", apparentée à un processus dynamique, tel qu'il était mis en œuvre, par exemple, lors du bain tinctorial.

Poursuivant l'exploration de la langue grecque, nous nous sommes mis en quête des mentions de couleur dans les textes. L'analyse de quelques exemples précis, en

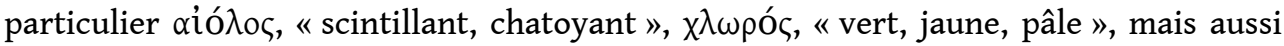

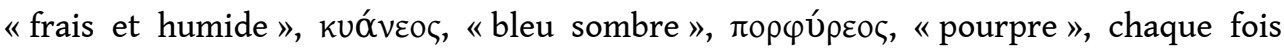
replacés dans leurs contextes d'emploi, a permis de faire jaillir leur riche polysémie et la prégnance de leur valeur affective. Elle a conduit à remettre en question la notion d'«adjectif de couleur", impropre lorsque l'on s'efforce de comprendre l'univers poétique archaïque. La perception que les Grecs avaient des couleurs reposait sur une intimité avec d'autres sensations que nous considérons comme distinctes: lumière, éclat et chatoiement, mouvement et vibration, matière et texture, voire même odeurs ou sonorités. Leur sensibilité chromatique passait d'abord par le truchement du matériau. À leurs yeux, les couleurs étaient indissociables de leur support, objet en métal, tissu teint ou sculpture peinte, et de l'usage social qui en était fait.

Portant alors attention aux gestes des artisans, aux matériaux - pigments et colorants nous nous sommes efforcée de reconstituer une histoire des pratiques polychromes et, à travers elles, de retrouver les formes de l'expérience sensible des Grecs relative aux couleurs. Par delà la grande variété des techniques, il semblerait que les méthodes employées par les artisans de la couleur aient convergé vers un même but : obtenir une couleur pure, qui ne soit pas altérée par un mélange, et, dotée d'un éclat intrinsèque, demeure vive et saturée ; une couleur stable, durable, qui échappe aux contingences du $\chi \rho \omega \omega \varsigma$, trop prompt à s'altérer. Les préoccupations qui s'exprimaient dans les gestes et les productions des artisans se retrouvaient en partie dans la voix des poètes et constituaient autant d'indices d'une sensibilité privilégiant l'alliance de couleurs franches, contrastées, vibrantes de lumière, dont l'éclat intense procurait le contentement du regard.

14 L'étape suivante de l'enquête a consisté à approfondir la dimension sociale de la couleur, afin de montrer que celle-ci agissait comme un signe opératoire, faisait office de critères d'identification et de reconnaissance dévoilant la place assignée à chacun à l'intérieur de la communauté. Les frontières colorées délimitaient des sphères d'identité, soulignaient l'existence de statuts sociaux distincts, balisant l'espace du vécu et marquant les distances du centre aux marges. Le chromatisme fournissait un modèle pour concevoir l'altérité. Femmes au teint "blanc », c'est-à-dire délicat et éclatant ; vieillards chenus; étrangers à la peau noire, brûlée par le soleil, ou roux aux yeux bleus, comme les fauves : tous portaient sur leur corps les stigmates de leur différence. La couleur pouvait aussi se muer en signe d'excellence : au sommet d'une hiérarchie chromatique, possédant des implications sur les plans politique et social, trônaient les couleurs " belles et bonnes ", conformes à l'idéal aristocratique. Il s'agissait de couleurs fortement saturées et éclatantes, qui conféraient à celui qui les arborait une aura supérieure, le plaçant au-dessus des autres individus. Elles s'incarnaient dans des 
matières, comme la pourpre et l'or, l'écarlate, la chevelure blonde, autant de sources de lumière capables de rivaliser avec l'éclat cosmique du divin et de susciter un plaisir

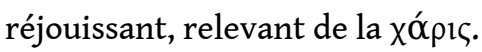

15 Élargissant le champ de l'investigation, nous nous sommes alors efforcée de restituer, dans un dernier temps, la structuration du réseau sémantique et affectif construit autour des signes chromatiques, au sein de l'imaginaire collectif des Grecs. Assurément, la complexité des systèmes symboliques mis en œuvre par les couleurs ne se réduisait pas à une polarité entre le blanc et le noir, la lumière et l'obscurité, même s'il est vrai que la sensibilité grecque appréciait une forme d'équilibre reposant sur une tension entre le pôle de la blancheur et celui de la noirceur. L'interprétation du rôle joué par les couleurs, lors des rites de passage ou du culte rendu aux dieux, requiert donc la plus grande prudence et un examen scrupuleux du contexte d'énonciation. La question de l'agencement des couleurs, en vue d'une composition harmonieuse, nous a alors conduite vers la notion antique de poikilon, qui gagne à être confrontée à celle, moderne, de polychromie. Pour clore la recherche, nous nous sommes donc employée à dresser un inventaire, une cartographie de la configuration sémantique et affective de

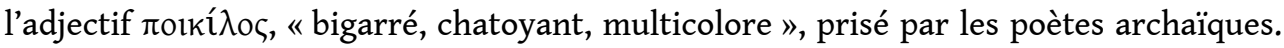
Le mot désignait un objet finement ouvré, dont le rayonnement polychrome reposait sur l'alliance harmonieuse de plusieurs matériaux, mais aussi le jeu fascinant de surfaces mouvantes et composites, tel le plumage des oiseaux, les écailles rugueuses $\mathrm{du}$ serpent, ou encore des instruments de séduction qui relevaient de l'éros, tenaient de la magie et trouvaient parfois à s'exprimer dans le chant mélodieux du poète.

L'étude menée dans le cadre de ce travail a ainsi dévoilé la coexistence, dans la Grèce archaïque, de deux façons distinctes et complémentaires de concevoir et d'appréhender la couleur : celle qui passait par l'analogie avec la peau, le $\chi \rho \omega ́ c$, à savoir une surface unie et «fleurie »; celle qui consistait à l'envisager sous le prisme de la diversité, de la pluralité, avec pour modèle le poikilon, notion qui renvoie aux jeux de contrastes chromatiques et aux amalgames de matières. Les deux facettes du chromatisme se retrouvaient dans le domaine de la sculpture : au type du kouros, jeune homme au corps nu enduit d'ocre, irradiant d'un rouge-brun intense, répondait la korè au vêtement chatoyant, rehaussé de motifs bigarrés. En somme, deux visages pour la couleur : l'un masculin, l'autre féminin.

La recherche a également permis de mettre en relief la spécificité de la culture chromatique grecque archaïque et sa perméabilité aux influences étrangères. Elle se perçoit dans les changements qui ont affecté les arts visuels, en particulier la diffusion des diverses pratiques polychromes, à partir du début du viI ${ }^{e}$ siècle - éclosion du style céramique dit "polychrome", développement de l'architecture en pierre et de la sculpture monumentale destinés à recevoir un traitement coloré, naissance de la " grande " peinture. Stimulés par les contacts noués avec les civilisations d'Égypte et du Proche-Orient et la découverte des objets richement décorés qui en provenaient, ces changements témoignent de l'éclosion d'une sensibilité tournée vers la bigarrure, qui

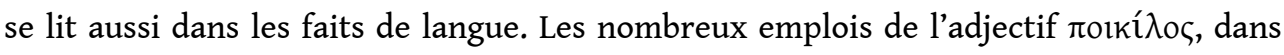
les textes de l'époque archaïque, l'attestent. Le chatoiement des couleurs occupait une place centrale dans les cités archaïques : comment comprendre, sinon, que la mitre multicolore de Lydie, dont Sappho et Pindare vantaient les charmes ${ }^{18}$, soit devenue la bannière diaprée dont les aristocrates souhaitaient conserver l'apanage? Il nous faut admettre que la bigarrure était alors une marque raffinée de prestige, un mode de 
reconnaissance sociale, non le signe d'une culture populaire frustre et primitive, comme l'estimait Henri Lechat, se faisant le porte-parole d'une tradition hostile à la couleur, née en Europe au XVII siècle lors de la "querelle du coloris ${ }^{19}$," et encore vivace, à bien des égards, aujourd'hui.

\section{NOTES}

1. Les premières remarques concernant les particularités du lexique chromatique grec figurent dans le Traité des couleurs (Zur Farbenlehre) de GOETHE, paru en 1810.

2. P. VEYNE, L'inventaire des différences, Paris, Seuil, 1976, p. 52-53.

3. Cette thèse de doctorat, préparée sous la direction de Pascal PAYEN, professeur d'Histoire grecque à l'Université de Toulouse-Le Mirail, s'intitule "Histoire du paysage sensible des Grecs à l'époque archaïque : le problème des couleurs" et a été soutenue le 18 novembre 2006.

4. W. GLADSTONE, "Homer's perception and use of colour", Studies on Homer and Homeric Age, Oxford, The University Press, 1858, III, p. 457-499 ; H. MAGNUS, Die geschichtliche Entwicklung des Farbensinnes, Leipzig, 1877, trad. fr. Histoire de l'évolution du sens des couleurs, Paris, Reinwald, 1878. 5. Diverses études ont été consacrées au débat qui a agité le milieu savant européen au cours du XIX siècle. Parmi celles-ci, on peut citer A. PRATER, The Rediscovery of Colour in Greek Architecture and Sculpture ", in M.A. TIVERIOS et D.S. TSIAFAKIS, Color in Ancient Greece. The Role of Color in Ancient Greek Art and Architecture 700-31 B.C., Thessalonique, Aristotle University of Thessaloniki, Lambrakis Research Foundation, 2002, p. 23-36. Nous avons également consacré un article à cette question: "Couleur et esthétique classique au XIX ${ }^{\mathrm{e}}$ siècle: l'art grec antique pouvait-il être polychrome? ", Íthaca. Quaderns Catalans de Cultura Classica 21 (2005), p. 146-157.

6. L'ouvrage qui a posé les fondements de l'idéal classique est sa Geschichte der Kunst des Altertums, Dresde, 1764, rééd. Darmstadt, 1982.

7. H. LECHAT, “ Observations sur les statues archaïques de type féminin du musée de l'Acropole ”, Bulletin de Correspondance Hellénique 14 (1890), p. 556.

8. L. GERNET, "Dénomination et perception des couleurs chez les Grecs", in I. MEYERSON (éd.), Problèmes de la couleur. Exposés et discussions du colloque du centre de recherche et de psychologie comparative tenu à Paris les 18, 19, 20 mai 1954, Paris, SEVPEN, 1957, p. 316.

9. C'est dans cette perspective que l'on peut situer les études rassemblées dans l'ouvrage dirigé par S. BETA et M.M. SASSI, I colori nel mondo antico : esperienze linguistiche e quadri simbolici, Fiesole, Cadmo, 2003.

10. A. CORBIN, Le territoire du vide. L'Occident et le désir de rivage 1500-1840, Paris, Aubier, 1988, p. 7.

11. Citons A. CORBIN, Les cloches de la terre. Paysage sonore et culture sensible dans les campagnes au XIXe siècle, Paris, Albin Michel, 1994 ; Le miasme et la jonquille. L'odorat et l'imaginaire social, XVIII ${ }^{e}-\mathrm{XIX}{ }^{e}$ siècles, Paris, Aubier, rééd. Flammarion, 1986.

12. M. Pastoureau, "La couleur et l'historien ", in Pigments et colorants de l'Antiquité et du Moyen Âge. Peinture, enluminure : études historiques et physico-chimiques, Paris, Éd. du CNRS, 1990, p. 29-30.

13. Plusieurs ouvrages, récemment parus, ont montré l'intérêt d'« une approche diversifiée des phénomènes de la couleur dans l'Antiquité par une lecture croisée des textes littéraires, philosophiques et techniques évalués à la lumière des nouvelles données scientifiques et archéologiques » : on citera notamment A. ROUVERET, S. DUBEL et V. NAAS (dir.), Couleurs et matières 
dans l'Antiquité. Textes, techniques, pratiques, Paris, Éditions rue d'Ulm, 2006 (la citation est d'A. Rouveret, p. 8) et L. CLELAND et K. STEARS, Colour in the Ancient Mediterranean World, Oxford, John and Erica Hedges, 2004.

14. Les vestiges de la peinture archaïque (pinakes et peintures murales) sont nettement plus rares.

15. De remarquables progrès ont été réalisés dans de domaine, grâce de nouvelles méthodes d'analyse fiables expérimentées par B. BOURGEOIs et Ph. JOCKEY sur les statues hellénistiques de Délos (nous renvoyons à leur article "Approches nouvelles de la polychromie des sculptures hellénistiques de Délos", Comptes rendus de l'Académie des Inscriptions et Belles Lettres, 2001, p. 629-665). En revanche, aucun projet similaire n'a été entrepris pour le dossier de la sculpture archaïque, en dépit des travaux menés par V. BRINKMANN (Die Polychromie der archaischen und frühklassischen Skulptur, Munich, Biering and Brinkmann, 2003). Sur les efforts de reconstitution de la polychromie des œuvres antiques, on se reportera également au catalogue de l'exposition de Munich : V. BRINKMANN, R. wÜNSCHE, U. wURNIG (dir.), Bunte Götter, die Farbigkeit antiker Skulptur, Munich, Staatliche Antikensammlungen und Glyptothek, 2004.

16. L. FEBVRE, “La sensibilité et l'histoire. Comment reconstituer la vie affective d'autrefois? ”, Annales d'histoire sociale 3 (1941), p. 12.

17. L. GERNET, "Dénomination et perception des couleurs chez les Grecs", p. 315. L'anecdote relatée par Ion de Chios a été conservée par Athénée (Deipnosophistes, XIII, 603 e-604d = Ion de Chios, FGrHist 392 F 6).

18. Sappho, fr. 98a, 10-11 ; fr. 98b, 1-3 Lobel-Page ; Pindare, Néméennes, VIII, 15 ; fr. 179, 1-2 Maehler.

19. Sur la querelle et ses racines antiques, voir J. LICHTENSTEIN, La couleur éloquente. Rhétorique et peinture à l'âge classique, Paris, Flammarion, 1989, rééd. 1999.

\section{AUTEUR}

\section{ADELINE GRAND-CLÉMENT}

ATER en Histoire grecque à l'Université de Provence

adelinegc@yahoo.fr 